
\title{
25 Research Square \\ Using a Physician Survey to Estimate the Economic Burden of Fibromyalgia in China
}

\section{Wesley Furnback}

Elysia Group Ltd.

\section{Feifei Chen}

Pfizer Upjohn Medical Trading Co., Ltd.

Jim Li

Pfizer Inc.

Bruce CM Wang ( $\nabla$ bruce.wang@elysiagroup.com )

Elysia Group Ltd. https://orcid.org/0000-0002-2612-2853

\section{Dongfeng Liang}

Chinese PLA General Hospital

\section{Research article}

Keywords: Burden of illness, fibromyalgia, health economic model

Posted Date: October 5th, 2020

DOI: https://doi.org/10.21203/rs.3.rs-61741/v1

License: (c) (1) This work is licensed under a Creative Commons Attribution 4.0 International License. Read Full License 


\section{Abstract}

Background: Fibromyalgia (FM) is a chronic pain disorder with a global prevalence estimated to be between 2 and 3\%. In addition to the chronic pain incurred by patients, FM is commonly associated with comorbidities and complications such as depression, anxiety, and sleep disturbances. This study estimates the economic burden of patients with FM in China using a physician survey.

Methods: A burden of illness model was constructed using a micro-costing approach to estimate the direct cost associated with FM patients in China. FM-related comorbidities of anxiety, depression, and sleep disturbance were included in the model. Treatment utilization and costs for FM and FM-related comorbidities were included as well as FM-related healthcare resource utilization (physician visits, hospitalizations, blood tests, and radiologic tests). FM treatments included nonsteroidal antiinflammatory drugs, pregabalin, duloxetine, amitriptyline, tramadol, Chinese medicine, physiotherapy, and acupuncture. The model leveraged the results of a physician survey, which targeted 6 rheumatologists and pain experts each with 5-10 FM patients per month in China. All costs are presented in Renminbi ( $¥)$ using spot exchange rates as of May 1, 2020.

Results: From the physician survey, the prevalence rate of FM in China was estimated to be $2.8 \%$ with $75.8 \%$ as female. The economic model estimated the annual per patient direct medical cost of FM to be $¥ 17,377$. Within these costs, FM-medication and treatment costs $(¥ 11,216)$, healthcare resource utilization $(¥ 4,297)$, and costs for medications treating FM-related comorbidities ( $¥ 1,863)$ were the highest contributors. Healthcare resource utilization costs were driven by physician visits $(¥ 2,787)$ followed by radiographic tests ( $¥ 808)$, blood tests ( $¥ 508)$, and hospitalizations ( $¥ 194)$.

Conclusion: The prevalence and gender distribution of FM patients in China is similar to those of other countries. The economic model estimates patients with fibromyalgia in China to incur significant economic costs.

\section{Background}

Fibromyalgia (FM) is a chronic pain disorder affecting muscles and soft tissue while causing significant health and economic burden to patients [1]. The American College of Rheumatology (ACR) has published guidelines to define the criteria for diagnosing fibromyalgia in adult patients. However, in clinical practice in China, the majority $(>60 \%)$ of physicians are unfamiliar with the ACR guidelines. Due to this lack of familiarity, which is not only a factor in China but globally, it is estimated that most cases take over 2 years to diagnosis [2].

The overall prevalence of fibromyalgia globally is estimated to be between 2 and $3 \%$ [2-3]. It is more than three times as common in females as males [3]. Prevalence rates also vary significantly from study to study depending on the criteria and sample, with very few national studies being conducted. Patients with FM experience a high rate of comorbid conditions compared to their non-FM counterparts. Common comorbidities include sleep disturbance/insomnia, anxiety, and depression [6]. Rates of depressive 
symptoms and anxiety were positively correlated with FM severity. Sleep disturbance/insomnia was highly prevalent regardless of FM severity with a reported prevalence of $52.4 \%$ in mild patients, $71.4 \%$ in moderate patients, and $69.2 \%$ in severe patients. The average number of comorbid conditions among mild patients was 2.9 compared to 4.1 in moderate patients and 4.4 in severe patients [6].

In China, a study of 107 confirmed FM patients reported over $20 \%$ had lumbar disk herniation or suspected or misdiagnosed spondyloarthritis, and $14 \%$ had osteoarthritis. Furthermore, $12.15 \%$ had either anxiety or depression [7].

There currently is no cure for FM and the majority of Chinese rheumatologists believe the goal of treatment should be to focus on relieving symptoms [8]. In China the preferred treatments for FM patients cared for by rheumatologists include antidepressants (79.1\%), nonsteroidal anti-inflammatory drugs (NSAIDs) (61.2\%), physical therapy (56.0\%), and exercise (52.6\%). The majority of rheumatologists (95.3\%) were prescribing pharmacotherapy to patients with a focus on relieving symptoms of $F M$, while $71.8 \%$ believed there was no effective fibromyalgia therapy [8].

Patients with fibromyalgia have a significant economic and humanistic burden. In the United States, patients with fibromyalgia were founded to have costs that were three-times higher than their non-FM counterparts [9]. This trend in higher costs has held true in global studies as well, with heightened costs for FM patients [10-11]. The economic impact has been described as similar to rheumatoid arthritis [12]. In addition to the high economic burden patients also experience a considerable humanistic burden with lower rates of employment, reduced productivity, and lower quality of life compared to non-FM patients $[10,13,14]$.

The primary objective of this study was to estimate the financial burden of fibromyalgia in China through the development of an economic model. The secondary objective was to conduct a survey of physicians to understand the prevalence, severity, and treatment patterns of patients with FM in China.

\section{Methods}

\section{Model Overview}

A model was developed to estimate the direct costs associated with FM in China. The model utilized a micro-costing approach and considered costs related to FM medications, medications for FM-related comorbidities, physician visits, hospitalizations, blood tests, and radiologic tests. This type of model has been used in previous FM studies to estimate the burden of disease within a health system [15]. Data for the model was sourced from a physician survey, a literature review, and publically available data.

\section{Physician Survey}

In the absence of recent literature describing the local treatment patterns of patients with fibromyalgia in China, a survey was administered to Chinese rheumatologists and pain physicians currently treating patients with FM. Their responses were based on their personal impressions based on their experience. 
Since the average physician in China may not be familiar with FM, the physicians chosen had an average of 5-10 patients per month. The selected physicians regularly participated in advisory boards and patient education activities for a variety of stakeholders. The survey covered four areas including 1 ) epidemiology of FM; 2) FM-related treatment patterns; 3) treatment patterns for FM-related comorbidities; and 4) healthcare resource utilization and costs for FM patients. A total of six Chinese rheumatologists were targeted for the surveys, which was administered in-person/via phone. Ethics committee approval was not required because the data were based on the opinions of clinical experts without the aide of patient charts.

\section{Model Inputs}

The model inputs were sourced from a combination of the literature and the physician survey described above. Clinical inputs included the percentage of patients on FM-related medications/therapy (Table 1) and medication for FM-related comorbidities as reported in the physician survey. The cost per day of each included medication is based on publically-available information and can be provided upon request.

Table 1

Physician-Reported Treatment Distribution

\begin{tabular}{|lllll|}
\hline MEDICATION & POINT ESTIMATE & MEDIAN & \multicolumn{2}{l|}{ RANGE } \\
\cline { 5 - 5 } & & & LOW & HIGH \\
\hline NSAIDs & $25.4 \%$ & $17.5 \%$ & $0.0 \%$ & $80.0 \%$ \\
\hline Chinese Herbal / Patent Medicine & $18.3 \%$ & $15.0 \%$ & $0.0 \%$ & $50.0 \%$ \\
\hline Pregabalin & $67.1 \%$ & $62.5 \%$ & $50.0 \%$ & $90.0 \%$ \\
\hline Duloxetine & $43.3 \%$ & $45.0 \%$ & $20.0 \%$ & $60.0 \%$ \\
\hline Tramadol & $16.7 \%$ & $20.0 \%$ & $0.0 \%$ & $30.0 \%$ \\
\hline Amitriptyline & $13.5 \%$ & $7.5 \%$ & $0.0 \%$ & $55.0 \%$ \\
\hline Physiotherapy & $57.5 \%$ & $50.0 \%$ & $0.0 \%$ & $100.0 \%$ \\
\hline Acupuncture & $12.5 \%$ & $2.5 \%$ & $0.0 \%$ & $60.0 \%$ \\
\hline
\end{tabular}

Resource utilization included physician visits of 9.33 per year and 0.23 hospital days per year (physician survey). The cost per physician visit (¥298.72) and hospital day (¥841.90) was sourced from a recent burden of illness study for postherpetic neuralgia in China [16]. A cost per year for lab tests (¥508.33) and radiologic tests (¥808.33) was also applied to patients.

The model took a societal perspective and reported the direct cost per FM patient in China. All costs were reported in 2019 Renminbi ( $¥)$ using spot exchange rates as of May 1, 2020 (1 USD = ¥7.06). 


\section{Results}

\section{Physician Survey}

The response rate to the survey was $100 \%$ with all physicians providing completed surveys. The physicians were rheumatologists and pain physicians located in the following hospitals: two physicians were from the Chinese PLA General Hospital and one each was from the Beijing Tsinghua Chang Gung Hospital, Shanghai Ruijin Hospital, Harbin Medical University, and Shanghai Xinhua Hospital. The prevalence rate in the Chinese population was estimated to be $2.8 \%$ (Range: $1 \%-6 \%$ ) with $75.8 \%$ (Range: $70 \%-87 \%$ ) of FM patients being female. The severity of FM within patients was fairly evenly distributed with an estimated $32.2 \%$ having mild $\mathrm{FM}, 38.1 \%$ with moderate $\mathrm{FM}$, and $29.7 \%$ with severe $\mathrm{FM}$. All physicians estimated that $100 \%$ of diagnosed patients across all severities were treated for FM.

Physician-reported rate of treatment for each class of medication is shown in Table 1. Pregabalin (67.1\%), and duloxetine (43.3\%) were the most frequently utilized pharmacological treatments followed by NSAIDs ( $25.4 \%)$, and Chinese medicine (18.3\%). A significant percentage of patients were also undergoing physiotherapy (57.5\%), and acupuncture (12.5\%).

The distribution of specific NSAIDs were $84.7 \%$ for celecoxib ( $40 \%$ on $200 \mathrm{mg} /$ day and $60 \%$ on $400 \mathrm{mg} /$ day), $6.7 \%$ for etoricoxib (60 mg/day), $6.7 \%$ for meloxicam ( $15 \mathrm{mg} /$ day), and $2.0 \%$ for loxoprofen (90 mg/day).

It was estimated that $36.7 \%$ of patients on pregabalin received $150 \mathrm{mg} /$ day and the remaining $63.7 \%$ received $300 \mathrm{mg} /$ day. The majority (89.2\%) of patients on duloxetine received $60 \mathrm{mg} /$ day, while $8.3 \%$, $1.7 \%$, and $0.8 \%$ received 30,90 , and $120 \mathrm{mg} /$ day, respectively.

Patients receiving tramadol were mostly on a dose of $100 \mathrm{mg} /$ day $(70.0 \%)$ with $20.0 \%$ receiving $50 \mathrm{mg} /$ day and $10.0 \%$ receiving $150 \mathrm{mg} /$ day. Amitriptyline was most commonly utilized at either $12.5 \mathrm{mg} /$ day or $25 \mathrm{mg} /$ day ( $40.0 \%$ of patients for both dose), while $20.0 \%$ received $18.8 \mathrm{mg} /$ day.

Surveyed physicians estimated one-third (range: $0 \%-90 \%$ ) of FM patients received medication to treat anxiety, $8.8 \%$ (range: $0.0 \%-30.0 \%$ ) to treat depression, and $36.7 \%$ (range: $0.0 \%-90.0 \%$ ) to treat a sleeping disturbance. Of patients receiving medication for anxiety, lorazepam was the most commonly used with $73.8 \%$ of patients. The remaining patients were receiving $12.5 \mathrm{mg} /$ day of clonazepam $(25.0 \%)$ or $1.2 \mathrm{mg} /$ day of alprazolam (1.3\%). Patients receiving depression medication received venlafaxine most frequently $(41.7 \%)$ followed by flupentixol/melitracen (30.0\%), citalopram (25.0\%), and sertraline (3.3\%). Sleep disturbance was most frequently treated with olanzapine (76.3\%) followed by lorazepam (23.8\%). The distribution of dosing for each medication is shown in Table 2. 
Table 2

Distribution of Dosing of Medications for FM-Related Comorbidities

\begin{tabular}{|ll|}
\hline MEDICATION & POINT ESTIMATE \\
\hline Depression Medication & \\
\hline Venlafaxine & \\
\hline $112.5 \mathrm{mg} /$ day & $80.0 \%$ \\
\hline $150 \mathrm{mg} /$ day & $20.0 \%$ \\
\hline Citalopram & \\
\hline $10 \mathrm{mg} /$ day & $66.7 \%$ \\
\hline $15 \mathrm{mg} /$ day & $33.3 \%$ \\
\hline Flupentixol/melitracen & \\
\hline $1.5 \mathrm{mg} /$ day & $44.4 \%$ \\
\hline $2 \mathrm{mg} /$ day & $55.6 \%$ \\
\hline Sertraline & \\
\hline $50 \mathrm{mg} /$ day & $100 \%$ \\
\hline Sleep Disturbance Medication & \\
\hline Lorazepam & $32.9 \%$ \\
\hline $0.75 \mathrm{mg} /$ day & $32.8 \%$ \\
\hline Olanzapine & \\
\hline 2 mg / day & \\
\hline $2.5 \mathrm{mg} /$ day & \\
\hline $3.5 \mathrm{mg} /$ day & \\
\hline $5 \mathrm{mg} / \mathrm{day}$ & \\
\hline Anxiety Medication & \\
\hline Alprazolam & \\
\hline $1.2 \mathrm{mg} /$ day & \\
\hline Lorazepam & \\
\hline $0.75 \mathrm{mg} /$ day & \\
\hline $1.0 \mathrm{mg} /$ day & \\
\hline
\end{tabular}




\begin{tabular}{|ll|}
\hline MEDICATION & POINT ESTIMATE \\
\hline Clonazepam & \\
$12.5 \mathrm{mg} /$ day & $100 \%$ \\
\hline
\end{tabular}

FM-related healthcare resource utilization was also collect in the surveys. Two-thirds of physicians reported FM patients had physician visits once every one to three months, and the other third of physicians reported visits once per month. The average cost of a physician visit was reported to be $¥ 159$ per visit.

\section{Burden of Illness}

The estimated total cost per patient per year for an FM patient was $¥ 17,377$. These costs were driven by medications for FM with $¥ 11,216$ (64.5\%) followed by healthcare resource utilization $(¥ 4,297 ; 10.7 \%)$ and medication costs for treating FM-related comorbidities (¥1,863; 24.8\%) (Fig. 1).

Within FM medication costs the largest contributors to costs were pregabalin $(¥ 7,054 ; 62.9 \%)$, and duloxetine $(¥ 2,525 ; 22.5 \%)$ followed by NSAIDs ( $¥ 711 ; 6.3 \%)$, Chinese medicine $(¥ 685 ; 6.1 \%)$, tramadol $(¥ 237 ; 2.1 \%$ ), and amitriptyline ( $¥ 6 ; 0.1 \%)$. Costs for medications treating FM-related comorbidities were highest for sleep disturbance ( $¥ 1,528 ; 82.0 \%)$ followed by depression ( $¥ 264 ; 14.2 \%)$, and anxiety ( $¥ 71$; $3.8 \%)$. Healthcare resource utilization $(¥ 4,297)$ included physician visits $(¥ 2,787 ; 64.9 \%)$, radiological tests (¥808; 18.8\%), lab tests (¥508;11.8\%), and hospitalizations ( $¥ 194 ; 4.5 \%)$.

\section{Discussion}

Patients with FM in China incur significant direct costs as a result of their condition. Per patient direct costs of $¥ 17,377$ were driven by FM medication and healthcare resource utilization. To the authors' knowledge, this is the first estimation of the economic burden of FM patients in China.

Globally the economic impact of fibromyalgia has been extensively reported. While the difference in health systems and costs makes a direct comparison between the results from this analysis to analyses in other health settings difficult, the drivers of costs can be compared. The exact prevalence and incidence of FM in China are largely unknown. There have been few studies attempting to estimate the prevalence in different regions and cities, and no studies estimating nationally [4-5]. A 2015 study evaluating the prevalence of FM in a small sample of 4,056 residents of Shantou estimated a prevalence rate of $0.12 \%$ [4]. A similar study conducted in Hong Kong reported a prevalence of $0.82 \%$ [5].

In Taiwan, the direct cost per year for patients with FM was estimated to be $¥ 14,000$. FM-related medications were responsible for $56.6 \%$ ( $¥ 7585)$ of costs, resource utilization was $14.1 \%$ ( $¥ 1885$ ), and comorbid medication was due to $29.2 \%$ of costs [15] A study of Japanese patients with FM determined 
the per-patient direct costs were $¥ 126,500$, which were significantly higher than a matched cohort of nonFM patients [13].

The average annual direct cost per FM patient in the United States with a diagnosis of FM diagnosis during the period of $2001-2004$ was $¥ 77,000$. Direct costs were driven by outpatient pharmaceuticals (28.8\%), inpatient stays (14.8\%), and physician visits (7.6\%) [12]. A study in the United States compared the costs and healthcare resource utilization of patients with FM based on severity. Of all the direct costs to the payer, $76.2 \%$ were for prescription medications in mild patients compared to $62.1 \%$ and $62.2 \%$ in moderate and severe patients, respectively [6].

Other economic burden studies have been conducted in China for other chronic disease areas. A 2019 publication estimated the economic burden of postherpetic neuralgia in China using a similar method. The study found total direct medical costs of $¥ 10,600$. The costs were driven by higher hospitalization costs of $¥ 9300$ compared to our study, which was driven mostly by higher drug costs [16]. The societal costs of patients with rheumatoid arthritis across 21 tertiary care hospitals between July 2009 and December 2010 were studied [17]. The total societal cost per patient-year was estimated to be $¥ 27,000$, of which $90 \%(¥ 24,300)$ were direct costs. The primary driver $(>50 \%)$ of costs were drug costs, which was similar to our analysis [17]. Patients with diabetes were reported to have total annual medical costs of $¥ 19,600$ and diabetes-related costs of $¥ 13,100$, which was closely correlated with our estimated annual direct cost of $¥ 17,377$ for FM patients [18].

The results of this analysis can be used by stakeholders in China to better understand the different sources of costs for FM patients in the healthcare system. While FM-related medications were the largest contributor to direct medical costs, the cost of treating comorbid conditions represented a significant $10.7 \%$ of direct medical costs. The optimal treatment selection to effectively treat FM and control comorbid conditions such as depression, anxiety, and sleep disturbance, could result in a reduction in treatment costs and healthcare resource utilization. Lastly, physician education is an important component of the effectiveness of treating FM. Physicians understanding the nature of the disease are able to more effectively treat it through the selection of the correct medication(s) for each individual patient and thus improve patient outcomes and perhaps lower the indirect costs associated with FM. Furthermore, treatments could include exercise and other healthy habits with minimal cost on the payer system.

This study has several limitations. First, the model included an average patient and the treatment patterns were heavily influenced by the physician survey. Within China, access to certain medications and the prices of medications can vary significantly, which would influence the cost of care. Second, this study may underestimate the economic burden of FM as it did not consider medications outside of FM medications and the three included comorbidities. There may be additional medications utilized by FM patients that were not covered. Additionally, the study only considered the costs of healthcare resource utilization for physician visits, lab tests, radiologic tests, and hospitalizations. FM patients likely utilize other healthcare services FM-related events. Third, the model did not consider the humanistic burden 
associated with FM, which is significant. Fourth, we did not include indirect costs in the study due to lack of data and reliable sources. Lastly, the model relied on data reported in the physician survey, which was made up of physicians with a relatively high caseload of FM patients. These results were based on physician impressions and not chart reviews, so one cannot determine whether the patients fulfilled any specific criteria for FM. Patients under the care of physicians without as much knowledge of FM may experience a different level of care. Moreover, there are limitations to generalizing the results of a survey based on 6 physicians to an entire country, such as China. The physicians do not represent the wideranging geographic regions of the country.

\section{Conclusion}

The survey results suggest the prevalence and gender distribution of FM patients in China to be similar to those of other countries. Patients with fibromyalgia have a significant economic burden on the Chinese health care system. Medications and lifestyle changes to better control FM could potentially reduce costs.

\section{Abbreviations}

ACR - American College of Rheumatology

FM - Fibromaylgia

NSAIDs - Nonsteroidal anti-inflammatory drugs

PLA - People's Liberation Army

\section{Declarations}

\section{Ethics approval and consent to participate:}

Ethics committee approval was not required because the data were based on the opinions of clinical experts without the aide of patient charts. No individual, patient-level data was referenced for the work. Please see the following link to support this statement: https://www.bu.edu/researchsupport/compliance/human-subjects/determining-if-irb-approval-is-needed/

\section{Consent for Publication:}

Not Applicable

\section{Availability of data and materials:}

Data sharing is not applicable to this article as no datasets were generated or analyzed during the current study.

\section{Competing interests:}


Bruce CM Wang, Wesley E Furnback, and Dongfeng Liang are paid consultants of Pfizer Upjohn Medical Trading Co., Ltd. Feifei Chen is an employee of Pfizer Upjohn Medical Trading Co., Ltd and Jim Z Li is an employee of Upjohn Division, Pfizer Inc.

\section{Funding:}

This study (including all publication fees) was funded by Pfizer Investment Co. Ltd.

\section{Author's Contributions:}

BW and WF provided data analysis, interpretation of data, and drafting of the work. FC and JL contributed to the study conception, design of work, and drafting of the work. DL contributed to the design of the work, data acquisition, and substantial revisioin of the draft.

\section{Acknowlegements:}

None

\section{References}

1. Wolfe F, Clauw DJ, Fitzcharles MA, et al. "2016 Revisions to the 2010/2011 fibromyalgia diagnostic criteria." Seminars in Arthritis and Rheumatism 2016; 46.3: 319-329.

2. Macfarlane GJ, Kronisch C, Dean LE, et al. "EULAR revised recommendations for the management of fibromyalgia." Annals of the Rheumatic Diseases 2017; 76.2: 318-328.

3. Queiroz LP. "Worldwide epidemiology of fibromyalgia." Current Pain and Headache Reports 2013; 17.8: 356.

4. Zeng SY, Gong Y, Zhang YP, et al. "Changes in the Prevalence of Rheumatic Diseases in Shantou, China, in the Past Three Decades: a COPCORD study." PloS one 2015; 10.9: e0138492.

5. Scudds RA, Li EKM, and Scudds RJ. "The prevalence of fibromyalgia syndrome in Chinese people in Hong Kong." Journal of Musculoskeletal Pain 2006; 14.2: 3-11.

6. Chandran A, Schaefer C, Ryan K, Baik R, McNett M, and Zlateva G. "The comparative economic burden of mild, moderate, and severe fibromyalgia: results from a retrospective chart review and cross-sectional survey of working-age US adults." Journal of Managed Care Pharmacy 2012; 18.6: 415-426.

7. Zhang Y, Liang D, Jiang R, et al. "Clinical, psychological features and quality of life of fibromyalgia patients: a cross-sectional study of Chinese sample." Clinical Rheumatology 2018; 37.2: 527-537.

8. Mu R, Li C, Zhang JX, et al. "National survey of knowledge, attitude and practice of fibromyalgia among rheumatologists in China." International Journal of Rheumatic Diseases 2013; 16.3: 258-263.

9. Berger A, Dukes E, Martin S, Edelsberg J, Oster G. Characteristics and healthcare costs of patients with fibromyalgia syndrome. International Journal of Clinical Practice 2007; 61.9: 1498-508. 
10. Lacasse $A$, Bourgault $P$, and Choinière $M$. "Fibromyalgia-related costs and loss of productivity: a substantial societal burden." BMC Musculoskeletal Disorders 2016; 17.1: 168.

11. Spaeth M. "Epidemiology, costs, and the economic burden of fibromyalgia." Arthritis Research \& Therapy 2009; 11.3: 117.

12. Silverman S, Dukes EM, Johnston SS, Brandenburg NA, Sadosky A, Huse DM."The economic burden of fibromyalgia: comparative analysis with rheumatoid arthritis." Current Medical Research and Opinion 2009; 25.4: 829-840.

13. Lee LK, Ebata N, Hlavacek P, DiBonaventura M, Cappelleri JC, and Sadosky A. "Humanistic and economic burden of fibromyalgia in Japan." Journal of Pain Research 2016; 9: 967.

14. Robinson RL, Kroenke K, Williams DA, et al. "Treatment patterns for patients with fibromyalgia: Baseline findings from the REFLECTIONS study." The Journal of Pain 2011; 12.4: P49.

15. Wang BCM, Furnback WE, Ney JP, Garrison LP, Fang C, and T-Form Group. "THU0511 Estimating the Burden of Illness of Fibromyalgia in Taiwan." Annals of the Rheumatic Diseases 2013; 72.Suppl 3: A336-A337.

16. Yu SY, Fan BF, Yang F, et al. "Patient and economic burdens of postherpetic neuralgia in China." ClinicoEconomics and Outcomes Research 2019; 11: 539-550.

17. Xu C, Wang X, Mu R, et al. " Societal costs of rheumatoid arthritis in China: a hospital-based crosssectional study." Arthritis Care \& Research 2014; 66.4: 523-531.

18. Huang Y, Vemer P, Zhu J, Postma MJ, and Chen W. "Economic burden in Chinese patients with diabetes mellitus using electronic insurance claims data." PLoS One 2016; 11.8: e0159297.

\section{Figures}

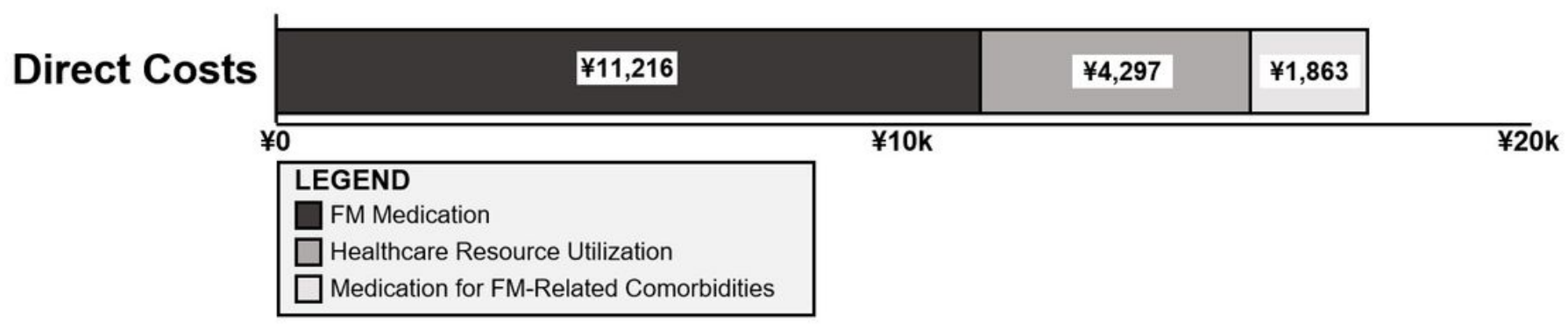

Figure 1

Direct Costs Per FM Patient

\section{Supplementary Files}

This is a list of supplementary files associated with this preprint. Click to download. 
- PhysicianSurvey.docx

- SummarySlideResubmission.docx 